\title{
CHARACTERISTIC EQUATIONS OF THE STANDARD AND DESCRIPTOR LINEAR ELECTRICAL CIRCUITS OF INTEGER AND FRACTIONAL ORDERS
}

\author{
Tadeusz KACZOREK ${ }^{*}$
}

${ }^{*}$ Faculty of Electrical Engineering, Białystok University of Technology, Wiejska 45D, 15-351 Białystok

kaczorek@isep.pw.edu.pl

received 14 June 2017, revised 5 September 2018, accepted 10 September 2018

\begin{abstract}
The problem of calculation of the characteristic equations of the standard and descriptor linear electrical circuits of integer and fractional orders is addressed. It is shown that the characteristic equations of standard and descriptor linear electrical circuits are independent of the method used in their analysis: the state space method, the mesh method and the node method. The considerations are illustrated by examples of standard and fractional linear electrical circuits.
\end{abstract}

Key words: Characteristic Equation, Standard, Descriptor, Fractional, Linear, Electrical Circuit

\section{INTRODUCTION}

A dynamical system is called positive if its trajectory starting from any nonnegative initial state remains forever in the positive orthant for all nonnegative inputs. An overview of state of the art in positive theory is given in the monographs (Farina and Rinaldi, 2000; Kaczorek, 2002). Variety of models having positive behavior can be found in engineering, especially in electrical circuits (Kaczorek and Rogowski, 2015), economics, social sciences, biology and medicine, etc. (Farina and Rinaldi, 2000; Kaczorek, 2002). The analysis of linear systems and electrical circuits has been addressed in (Antsaklis and Michel, 2006; Cholewicki, 1967; Kaczorek, 1992; Kaczorek and Rogowski, 2015; Kailath, 1980; Rosenbrock, 1970; Wolovich, 1974; Żak, 2003).

The mathematical fundamentals of fractional calculus are given in the monographs (Oldham and Spanier, 1974; Ostalczyk, 2008, 2016; Podlubny, 1999). The fractional systems theory and its applications is presented in (Dzieliński et al., 2009; Kaczorek, 2008a, 2008b, 2009, 2010, 2011a, 2011b, 2012a, 2013a, 2014a; Kaczorek and Rogowski, 2015; Sajewski, 2016; Vinagre et al., 2002).

The positive electrical circuits have been analyzed in (Kaczorek, 2011c, 2013b, 2014b, 2015a, 2015b, 2016; Kaczorek and Rogowski, 2015). The constructability and observability of standard and positive electrical circuits has been addressed in (Kaczorek, 2013b), controllability and observability in (Kaczorek, 2011d), the decoupling zeros in (Kaczorek, 2014b) and minimalphase positive electrical circuits in (Kaczorek, 2016). A new class of normal positive linear electrical circuits has been introduced in (Kaczorek, 2015b). Positive fractional linear electrical circuits have been investigated in (Kaczorek, 2013a), positive linear systems with different fractional orders in (Kaczorek, 2010, 2011a) and positive unstable electrical circuits in (Kaczorek, 2012b). Zeroing of state variables in descriptor electrical circuits has been addressed in (Kaczorek, 2013c).

In this paper the problem of calculation of the characteristic equations of the standard and descriptor linear electrical circuits of integer and fractional orders will be analyzed.

The paper is organized as follows. In section 2 some preliminaries concerning positive electrical circuits of integer and fractional orders are recalled. The characteristic equations of integer order electrical circuits analyzed by the state space method, mesh method and node method are investigated in section 3. The same problem for descriptor electrical circuits is addressed in section 4 . The characteristic equations of fractional electrical circuits are analyzed in section 5 . Concluding remarks are given in section 6 .

The following notation will be used: $\mathfrak{R}$ - the set of real numbers, $\Re^{n \times m}$ - the set of $\mathrm{n} \times \mathrm{m}$ real matrices, $\Re_{+}^{n \times m}$ - the set of $n \times m$ real matrices with nonnegative entries and $\Re_{+}^{n}=\Re_{+}^{n \times 1}$, $M_{n}$ - the set of $n \times n$ Metzler matrices (real matrices with nonnegative off-diagonal entries), $I_{n}$ - the $n \times n$ identity matrix.

\section{PRELIMINARIES}

Consider the linear continuous-time electrical circuit described by the state equation:

$\dot{x}(t)=A x(t)+B u(t)$,

where $x(t) \in \Re^{n}, u(t) \in \Re^{m}$ are the state and input vectors and $A \in \Re^{n \times n}, B \in \Re^{n \times m}$. It is well-known (Antsaklis, 2006; Cholewicki, 1967; Kaczorek and Rogowski, 2015; Kailath, 1980; Rosenbrock, 1970; Wolovich, 1974; Żak, 2003) that any standard linear electrical circuit composed of resistors, coils, capacitors and voltage (current) sources can be described by the equation (1). Usually as the state variables $x_{1}(t), \ldots, x_{n}(t)$ (the components of the vector $x(t))$ the currents in the coils and voltages on the capacitors are chosen.

Definition 1. (Kaczorek and Rogowski, 2015) The electrical circuit (1) is called (internally) positive if $x(t) \in \mathfrak{R}_{+}^{n}$, for any initial condition $x(0) \in \mathfrak{R}_{+}^{n}$ and every $u(t) \in \Re_{+}^{m}, t \in[0,+\infty)$. 
Theorem 1. (Kaczorek and Rogowski, 2015) The electrical circuit (1) is positive if and only if:

$A \in M_{n}, B \in \mathfrak{R}_{+}^{n \times m}$.

The positive electrical circuit (1) for $u(t)=0$ is called asymptotically stable if:

$\lim _{t \rightarrow \infty} x(t)=0$ for all $x(0) \in \Re_{+}^{n}$.

Theorem 2. (Kaczorek and Rogowski, 2015) The positive electrical circuit (1) is asymptotically stable if and only if:

$\operatorname{Re} \lambda_{k}<0$ for $k=1, \ldots, n$ or equivalently $\frac{\pi}{2}<\arg \lambda_{k}<\frac{3 \pi}{2}$

where $\lambda_{k}$ is the eigenvalue of the matrix $A \in M_{n}$ and:

$\operatorname{det}\left[I_{n} \lambda-A\right]=\left(\lambda-\lambda_{1}\right)\left(\lambda-\lambda_{2}\right) \ldots\left(\lambda-\lambda_{n}\right)$.

The following Caputo definition of the fractional derivative will be used (Kaczorek, 2012a):

$D^{\alpha} f(t)=\frac{d^{\alpha}}{d t^{\alpha}} f(t)=\frac{1}{\Gamma(n-\alpha)} \int_{0}^{t} \frac{f^{(n)}(\tau)}{(t-\tau)^{\alpha+1-n}} d \tau$,

$n-1<\alpha \leq n \in N=\{1,2, \ldots\}$,

where $\alpha \in \Re$ is the order of fractional derivative, $f^{(n)}(\tau)=$ $\frac{d^{n} f(\tau)}{d \tau^{n}}$ and $\Gamma(x)=\int_{0}^{\infty} e^{-t} t^{x-1} d t$ is the gamma function.

Consider fractional electrical circuits composed of resistors, coils, capacitors and voltage (current) sources. Using the Kirchhoff's laws we may describe the transient states in the electrical circuits by state equations (Kaczorek, 2012a; Kaczorek and Rogowski, 2015):

$D^{\alpha} x(t)=A x(t)+B u(t), 0<\alpha \leq 1$,

where $x(t) \in \mathfrak{R}^{n}, u(t) \in \mathfrak{R}^{m}$ are the state and input vectors and $A \in \mathfrak{R}^{n \times n}, B \in \mathfrak{R}^{n \times m}$.

Theorem 3. (Kaczorek, 2012a) The solution of equation (7) is given by:

$x(t)=\Phi_{0}(t) x_{0}+\int_{0}^{t} \Phi(t-\tau) B u(\tau) d \tau, x(0)=x_{0}$,

where:

$\Phi_{0}(t)=E_{\alpha}\left(A t^{\alpha}\right)=\sum_{k=0}^{\infty} \frac{A^{k} t^{k \alpha}}{\Gamma(k \alpha+1)}$

$\Phi(t)=\sum_{k=0}^{\infty} \frac{A^{k} t^{(k+1) \alpha-1}}{\Gamma[(k+1) \alpha]}$

and $E_{\alpha}\left(A t^{\alpha}\right)$ is the Mittag-Leffler matrix function (Kaczorek, 2012a).

Definition 2. (Kaczorek, 2012a) The fractional system (7) is called the (internally) positive fractional system if and only if $x(t) \in \mathfrak{R}_{+}^{n}$, $t \geq 0$ for any initial conditions $x_{0} \in \mathfrak{R}_{+}^{n}$ and all inputs $u(t) \in$ $\mathfrak{R}_{+}^{m}, t \geq 0$.

Theorem 4. (Kaczorek, 2012a) The fractional system (7) is positive if and only if

$A \in M_{n}, B \in \mathfrak{R}_{+}^{n \times m}$.

\section{CHARACTERISTIC EQUATIONS OF INTEGER ORDER ELECTRICAL CIRCUITS}

\subsection{State space method}

In this method as the state variables the voltages on the capacitors and the currents in the coils are chosen. The linear electrical circuits are described by the state equation (1). The characteristic polynomial of the circuit is given by:

$p(s)=\operatorname{det}\left[I_{n} s-A\right]=s^{n}+a_{n-1} s^{n-1}+\ldots+a_{1} s+a_{0}$

and its characteristic equation by $p(s)=0$.

Example 1. Consider the electrical circuit shown in Fig. 1 with given resistances $R_{1}, R_{2}, R_{3}$, inductances $L_{1}, L_{2}$ and voltage sources $e_{1}, e_{2}$.

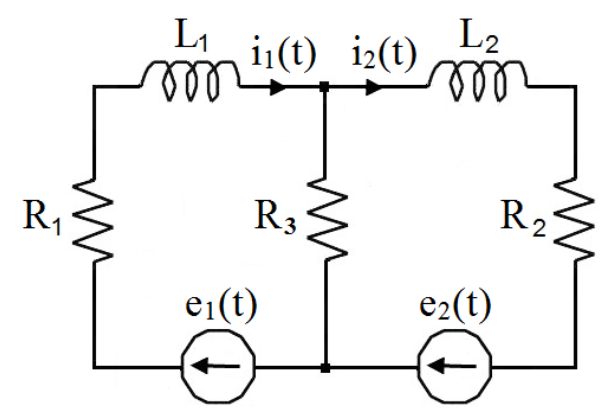

Fig. 1. Electrical circuit of Example 1

Using the Kirchhoff's laws for the electrical circuit we obtain the equations:

$e_{1}=R_{1} i_{1}+L_{1} \frac{d i_{1}}{d t}+R_{3}\left(i_{1}-i_{2}\right)$,

$e_{2}=R_{2} i_{2}+L_{2} \frac{d i_{2}}{d t}+R_{3}\left(i_{2}-i_{1}\right)$,

which can be written in the form:

$\frac{d}{d t}\left[\begin{array}{l}i_{1} \\ i_{2}\end{array}\right]=A_{1}\left[\begin{array}{l}i_{1} \\ i_{2}\end{array}\right]+B_{1}\left[\begin{array}{l}e_{1} \\ e_{2}\end{array}\right]$,

where:

$A_{1}=\left[\begin{array}{ll}-\frac{R_{1}+R_{3}}{L_{1}} & \frac{R_{3}}{L_{1}} \\ \frac{R_{3}}{L_{2}} & -\frac{R_{2}+R_{3}}{L_{2}}\end{array}\right], B_{1}=\left[\begin{array}{ll}\frac{1}{L_{1}} & 0 \\ 0 & \frac{1}{L_{2}}\end{array}\right]$.

The electrical circuit is positive since $A_{1} \in M_{2}$ and $B_{1} \in$ $\mathfrak{R}_{+}^{2 \times 2}$.

The characteristic equation of the electrical circuit has the form:

$\operatorname{det}\left[I_{2} s-A_{1}\right]=\left|\begin{array}{cc}s+\frac{R_{1}+R_{3}}{L_{1}} & -\frac{R_{3}}{L_{1}} \\ -\frac{R_{3}}{L_{2}} & S+\frac{R_{2}+R_{3}}{L_{2}}\end{array}\right|$
$=s^{2}+\left(\frac{R_{1}+R_{3}}{L_{1}}+\frac{R_{2}+R_{3}}{L_{2}}\right) s+\frac{R_{1}\left(R_{2}+R_{3}\right)+R_{2} R_{3}}{L_{1} L_{2}}=0$. 


\subsection{Mesh method}

Any linear electrical circuit composed of resistors, coils, capacitors and voltage (current) sources in transient states can be also analyzed by the use of the mesh method (Cholewicki, 1967; Kaczorek and Rogowski, 2015).

Using the mesh method and the Laplace transform for zero initial conditions we can describe the electrical circuit in transient states by the equation:

$Z(s) X(s)=E(s)$,

where: $X(s)=L[x(t)]=\int_{0}^{\infty} x(t) e^{-s t} d t \quad(L$ is the Laplace operator),

$Z(s)=\left[\begin{array}{lll}Z_{11}(s) & \cdots & Z_{1 n}(s) \\ \vdots & \ddots & \vdots \\ Z_{n 1}(s) & \cdots & Z_{n n}(s)\end{array}\right], E(s)=\left[\begin{array}{l}E_{1}(s) \\ \vdots \\ E_{n}(s)\end{array}\right]$.

Example 2. Using the mesh method and the Laplace transform for the electrical circuit (with given resistances $R_{1}, R_{2}, R_{3}$, inductances $L_{1}, L_{2}$ and voltage sources $e_{1}, e_{2}$ ) shown in Fig. 2 we obtain:

$\left[\begin{array}{ll}R_{1}+R_{3}+s L_{1} & -R_{3} \\ -R_{3} & R_{2}+R_{3}+s L_{2}\end{array}\right]\left[\begin{array}{l}I_{1}(s) \\ I_{2}(s)\end{array}\right]=\left[\begin{array}{l}E_{1}(s) \\ E_{2}(s)\end{array}\right]$

where $I_{k}(s)=\mathrm{L}\left[i_{k}(t)\right], E_{k}(s)=\mathrm{L}\left[e_{k}(t)\right], k=1,2$.

In this case we have:

$Z(s)=\left[\begin{array}{ll}R_{1}+R_{3}+s L_{1} & -R_{3} \\ -R_{3} & R_{2}+R_{3}+s L_{2}\end{array}\right], X(s)=\left[\begin{array}{l}I_{1}(s) \\ I_{2}(s)\end{array}\right]$,

$E(s)=\left[\begin{array}{l}E_{1}(s) \\ E_{2}(s)\end{array}\right]$.

Note that:

$\operatorname{det} Z(s)=\left|\begin{array}{ll}R_{1}+R_{3}+s L_{1} & -R_{3} \\ -R_{3} & R_{2}+R_{3}+s L_{2}\end{array}\right|=L_{1} L_{2} s^{2}$

$+\left[\left(R_{1}+R_{3}\right) L_{2}+\left(R_{2}+R_{3}\right) L_{1}\right] s+R_{1}\left(R_{2}+R_{3}\right)+R_{2} R_{3}$

and after multiplication by $\frac{1}{L_{1} L_{2}}$ we obtain:

$\operatorname{det} Z(s)=L_{1} L_{2} \operatorname{det}\left[I_{2} s-A_{1}\right]$.

From (19) we have the following conclusion.

Conclusion 1. The characteristic equation (15) of the electrical circuit can be also obtained by computation of the determinant of the matrix $Z(s)$ in the mesh method.

\subsection{Node method}

Any linear electrical circuit composed of resistors, coils, capacitors and voltage (current) sources in transient states can be also analyzed by the use of the node method. Using the node method and the Laplace transform for zero initial conditions we can describe the electrical circuit in transient states by the equation (Cholewicki, 1967; Kaczorek and Rogowski, 2015):

$Y(s) V(s)=I_{z}(s)$,

where:

$$
\begin{gathered}
Y(s)=\left[\begin{array}{lll}
Y_{11}(s) & \cdots & Y_{1 q}(s) \\
\vdots & \ddots & \vdots \\
Y_{q 1}(s) & \cdots & Y_{q q}(s)
\end{array}\right], V(s)=\left[\begin{array}{l}
V_{1}(s) \\
\vdots \\
V_{q}(s)
\end{array}\right], \\
I_{Z}(s)=\left[\begin{array}{l}
I_{z 1}(s) \\
\vdots \\
I_{z q}(s)
\end{array}\right],
\end{gathered}
$$

$q$ is the number of linearly independent nodes, $Y_{i j}(s)$ and $V_{i}(s)$, $i, j=1, \ldots, q$ are Laplace transforms of conductances and current sources of the electrical circuit, respectively.

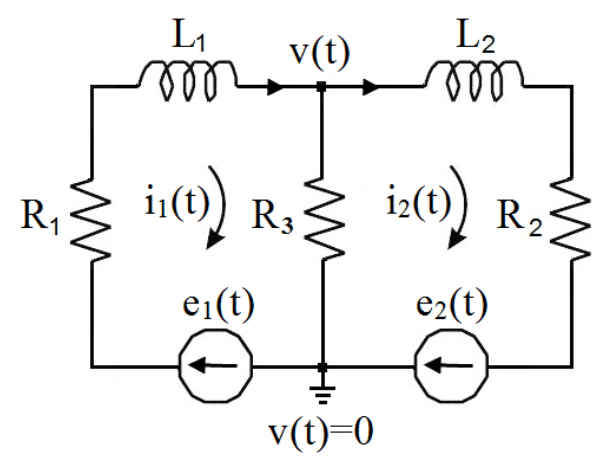

Fig. 2. Electrical circuit

Example 3. For the electrical circuit shown in Fig. 2 using the node method we obtain:

$Y(s) V(s)=I_{z}(s)$,

where $V(s)=\mathrm{L}[v(t)], E_{k}(s)=\mathrm{L}\left[e_{k}(t)\right], k=1,2$ and:

$Y(s)=Y_{11}(s)=\frac{1}{R_{1}+s L_{1}}+\frac{1}{R_{2}+s L_{2}}+\frac{1}{R_{3}}$,

$I_{z}(s)=\frac{E_{1}(s)}{R_{1}+s L_{1}}+\frac{E_{2}(s)}{R_{2}+s L_{2}}$.

Note that:

$\operatorname{det} Y(s)=Y(s)=\frac{1}{R_{1}+s L_{1}}+\frac{1}{R_{2}+s L_{2}}+\frac{1}{R_{3}}$

$=\frac{L_{1} L_{2} s^{2}+\left[\left(R_{1}+R_{3}\right) L_{2}+\left(R_{2}+R_{3}\right) L_{1}\right] s+R_{1}\left(R_{2}+R_{3}\right)+R_{2} R_{3}}{\left(R_{1}+s L_{1}\right)\left(R_{2}+s L_{2}\right) R_{3}}$

and after multiplication by $\frac{\left(R_{1}+s L_{1}\right)\left(R_{2}+s L_{2}\right) R_{3}}{L_{1} L_{2}}$, we obtain:

$\operatorname{det} Y(s)=\frac{L_{1} L_{2}}{\left(R_{1}+s L_{1}\right)\left(R_{2}+s L_{2}\right) R_{3}} \operatorname{det}\left[I_{2} s-A_{1}\right]$.

From (23) we have the following conclusion.

Conclusion 2. The characteristic equation (15) of the electrical circuit can be also obtained by computation of the determinant of the matrix $Y(s)$ in the node method.

In general case we shall prove the following theorem.

Theorem 5. The characteristic equation of any linear circuit composed of resistors, coils and capacitors is given in state equations method by:

$\operatorname{det}\left[I_{n} s-A\right]=s^{n}+a_{n-1} s^{n-1}+\ldots+a_{1} s+a_{0}=0$,

in mesh method by $\operatorname{det} Z(s)=0$ and in node method by $\operatorname{det} Y(s)=0$.

Proof. Applying the Laplace transform to (1) with zero initial conditions we obtain: 
$X(s)=\left[I_{n} s-A\right]^{-1} B U(s)=\frac{\left[I_{n} s-A\right]_{a d}}{\operatorname{det}\left[I_{n} s-A\right]} B U(s)$,

The equations (30) can be written in the form:

where $\left[I_{n} s-A\right]_{a d}$ is the adjoint matrix of $\left[I_{n} s-A\right]$.

From (16) we have:

$X(s)=Z^{-1}(s) E(s)=\frac{Z_{a d}(s)}{\operatorname{det} Z(s)} E(s)$.

Comparing the denominators of (25) and (26) we obtain that $\operatorname{det}\left[I_{n} s-A\right]=0$ is equivalent to $\operatorname{det} Z(s)=0$.

From (21a) we have:

$V(s)=Y^{-1}(s) I_{Z}(s)$.

Note that knowing $V(s)$ we can always find such matrix $P(s) \in \Re^{n \times q}(s)$ that:

$X(s)=P(s) V(s)$.

Substituting (27) into (28) we obtain:

$X(s)=P(s) Y^{-1}(s) I_{Z}(s)=\frac{P(s) Y_{a d}(s) I_{Z}(s)}{\operatorname{det} Y(s)}$.

Comparing the denominators of (25) and (29) we obtain that $\operatorname{det}\left[I_{n} s-A\right]=0$ is equivalent to $\operatorname{det} Y(s)=0$.

Remark 1. The characteristic polynomial and characteristic equation of any linear electrical circuit is independent of the voltage (current) sources. Therefore, computing the characteristic equation (polynomial) of the electrical circuit the voltage (current) sources can be assumed as zero.

Example 4. Consider the electrical circuit shown in Fig. 3 with given resistances $R_{k}, k=1, \ldots, 5$, inductances $L_{1}, L_{2}$, capacitance $C$ and source voltages $e_{1}, e_{2}$.

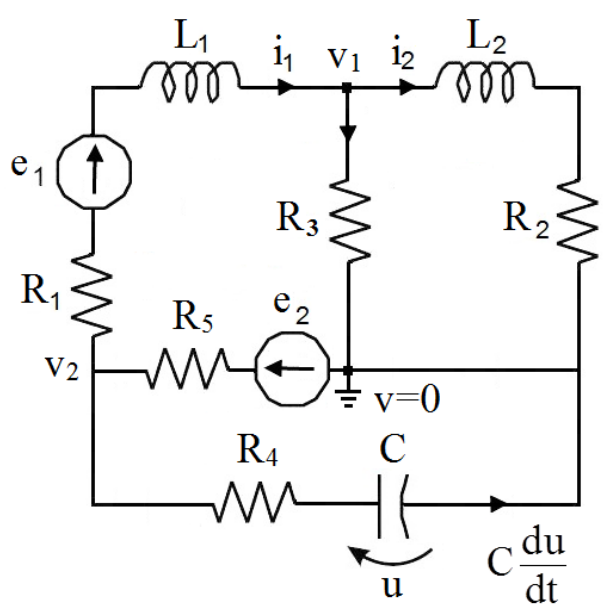

Fig. 3. Electrical circuit of Example 4

Using the Kirchhoff's laws for the electrical circuit we can write the equations:

$$
\begin{aligned}
e_{1}+e_{2} & =R_{11} i_{1}-R_{3} i_{2}+R_{5} C \frac{d u}{d t}+L_{1} \frac{d i_{1}}{d t}, \\
0 & =R_{22} i_{2}-R_{3} i_{1}+L_{2} \frac{d i_{1}}{d t}, \\
e_{2} & =u+R_{33} C \frac{d u}{d t}+R_{5} i_{1},
\end{aligned}
$$

where $R_{11}=R_{1}+R_{3}+R_{5}, R_{22}=R_{2}+R_{3}, R_{33}=R_{4}+R_{5}$.

$$
\begin{aligned}
& {\left[\begin{array}{lll}
L_{1} & 0 & R_{5} C \\
0 & L_{2} & 0 \\
0 & 0 & R_{33} C
\end{array}\right] \frac{d}{d t}\left[\begin{array}{l}
i_{1} \\
i_{2} \\
u
\end{array}\right]} \\
& =\left[\begin{array}{lll}
-R_{11} & R_{3} & 0 \\
R_{3} & -R_{22} & 0 \\
-R_{5} & 0 & -1
\end{array}\right]\left[\begin{array}{l}
i_{1} \\
i_{2} \\
u
\end{array}\right]+\left[\begin{array}{ll}
1 & 1 \\
0 & 0 \\
0 & 1
\end{array}\right]\left[\begin{array}{l}
e_{1} \\
e_{2}
\end{array}\right] .
\end{aligned}
$$

From (31) we have:

$$
\frac{d}{d t}\left[\begin{array}{l}
i_{1} \\
i_{2} \\
u
\end{array}\right]=A_{2}\left[\begin{array}{l}
i_{1} \\
i_{2} \\
u
\end{array}\right]+B_{2}\left[\begin{array}{l}
e_{1} \\
e_{2}
\end{array}\right],
$$

where:

$$
\begin{aligned}
A_{2}= & {\left[\begin{array}{lll}
L_{1} & 0 & R_{5} C \\
0 & L_{2} & 0 \\
0 & 0 & R_{33} C
\end{array}\right]^{-1}\left[\begin{array}{lll}
-R_{11} & R_{3} & 0 \\
R_{3} & -R_{22} & 0 \\
-R_{5} & 0 & -1
\end{array}\right] } \\
& =\left[\begin{array}{lll}
\frac{1}{L_{1}}\left(\frac{R_{5}^{2}}{R_{33}}-R_{11}\right) & \frac{R_{3}}{L_{1}} & \frac{R_{5}}{L_{1} R_{33}} \\
\frac{R_{3}}{L_{2}} & -\frac{R_{22}}{L_{2}} & 0 \\
-\frac{R_{5}}{R_{33} C} & 0 & -\frac{1}{R_{33} C}
\end{array}\right],
\end{aligned}
$$

$B_{2}=\left[\begin{array}{lll}L_{1} & 0 & R_{5} C \\ 0 & L_{2} & 0 \\ 0 & 0 & R_{33} C\end{array}\right]^{-1}\left[\begin{array}{ll}1 & 1 \\ 0 & 0 \\ 0 & 1\end{array}\right]=\left[\begin{array}{ll}\frac{1}{L_{1}} & \frac{1}{L_{1}}\left(1-\frac{R_{5}}{R_{33}}\right) \\ 0 & 0 \\ 0 & \frac{1}{R_{33} C}\end{array}\right]$.

Note that the electrical circuit is positive if and only if $R_{5}=0$.

The characteristic equation of the electrical circuit has the form:

$\operatorname{det}\left[I_{3} s-A_{2}\right]$

$$
\begin{aligned}
& =\left[\begin{array}{lll}
s+\frac{1}{L_{1}}\left(R_{11}-\frac{R_{5}^{2}}{R_{33}}\right) & -\frac{R_{3}}{L_{1}} & -\frac{R_{5}}{L_{1} R_{33}} \\
-\frac{R_{3}}{L_{2}} & s+\frac{R_{22}}{L_{2}} & 0 \\
\frac{R_{5}}{R_{33} C} & 0 & s+\frac{1}{R_{33} C}
\end{array}\right] \\
& =\frac{L_{1} L_{2} R_{33} C S^{3}+\left(L_{1} L_{2}-L_{2} R_{5}^{2} C+L_{2} R_{11} R_{33} C+L_{1} R_{22} R_{33} C\right) s^{2}}{L_{1} L_{2} R_{33} C} \\
& +\frac{\left(L_{1} R_{22}+L_{2} R_{11}-R_{22} R_{5}^{2} C-R_{33} R_{3}^{2} C+R_{11} R_{22} R_{33} C\right) s+R_{11} R_{22}-R_{3}^{2}}{L_{1} L_{2} R_{33} C} .
\end{aligned}
$$

Applying to the electrical circuit in Fig. 3 the mesh method we choose as the state variables the currents $i_{1}, i_{2}, C \frac{d u}{d t}$ and we obtain:

$Z(s)=\left[\begin{array}{lll}R_{11}+s L_{1} & -R_{3} & -R_{5} \\ -R_{3} & R_{22}+s L_{3} & 0 \\ -R_{5} & 0 & R_{33}+\frac{1}{s c}\end{array}\right]$,

where $R_{11}=R_{1}+R_{3}+R_{5}, R_{22}=R_{2}+R_{3}, R_{33}=R_{4}+R_{5}$ and:

$$
\begin{aligned}
& \operatorname{det} Z(S)=\frac{L_{1} L_{2} R_{33} C s^{3}+\left(L_{1} L_{2}-L_{2} R_{5}^{2} C+L_{2} R_{11} R_{33} C+L_{1} R_{22} R_{33} C\right) s^{2}}{S C} \\
& +\frac{\left(L_{1} R_{22}+L_{2} R_{11}-R_{22} R_{5}^{2} C-R_{33} R_{3}^{2} C+R_{11} R_{22} R_{33} C\right) s+R_{11} R_{22}-R_{3}^{2}}{s C} .
\end{aligned}
$$

From comparison of (33) and (35) it follows that the characteristic equations are equivalent.

Applying to the electrical circuit in Fig. 3 the node method we obtain: 
$Y(s)=\left[\begin{array}{ll}\frac{1}{R_{1}+s L_{1}}+\frac{1}{R_{2}+s L_{2}}+\frac{1}{R_{3}} & -\frac{1}{R_{1}+s L_{1}} \\ -\frac{1}{R_{1}+s L_{1}} & \frac{1}{R_{1}+s L_{1}}+\frac{1}{R_{4}+\frac{1}{s C}}+\frac{1}{R_{5}}\end{array}\right]$

and:

$\operatorname{det} Y(s)=\frac{L_{1} L_{2} R_{33} C s^{3}+\left(L_{1} L_{2}-L_{2} R_{5}^{2} C+L_{2} R_{11} R_{33} C+L_{1} R_{22} R_{33} C\right) s^{2}}{R_{3} R_{5}\left(s R_{4} C s+1\right)\left(R_{1}+s L_{1}\right)\left(R_{2}+s L_{2}\right)}$ $+\frac{\left(L_{1} R_{22}+L_{2} R_{11}-R_{22} R_{5}^{2} C-R_{33} R_{3}^{2} C+R_{11} R_{22} R_{33} C\right) s+R_{11} R_{22}-R_{3}^{2}}{R_{3} R_{5}\left(s R_{4} C s+1\right)\left(R_{1}+s L_{1}\right)\left(R_{2}+s L_{2}\right)}$,

where:

$R_{11}=R_{1}+R_{3}+R_{5}, R_{22}=R_{2}+R_{3}, R_{33}=R_{4}+R_{5}$.

From comparison of (33) and (37) if follows that the characteristic equations are equivalent.

\section{DESCRIPTOR ELECTRICAL CIRCUITS}

In this section the previous results will be extended to descriptor linear electrical circuits.

Consider the linear electrical circuit described by the equation:

$E \dot{x}=A x+B u$,

where $x=x(t) \in \mathfrak{R}^{n}, u=u(t) \in \mathfrak{R}^{m}$ are the state and input vectors and $E, A \in \mathfrak{R}^{n \times n}, B \in \mathfrak{R}^{n \times m}$. It is assumed that:

$\operatorname{det} E=0$ and $\operatorname{det}[E s-A] \neq 0$ for some $s \in \mathbf{C}$

(the field of complex numbers).

Definition 3. The linear electrical circuit described by (38) satisfying the assumption (39) is called a descriptor (singular) electrical circuit.

Theorem 6. Linear electrical circuit is descriptor if it contains at least one mesh consisting of only ideal capacitors and voltage sources or at least one node with branches with coils.

Proof. The proof is given in (Kaczorek and Rogowski, 2015).

Example 5. Consider the descriptor electrical circuit shown in Fig. 4 with given resistances $R_{k}, k=1,2,3$, inductances $L_{k}, k=$ $1,2,3$ and voltage sources $e_{1}, e_{2}$.

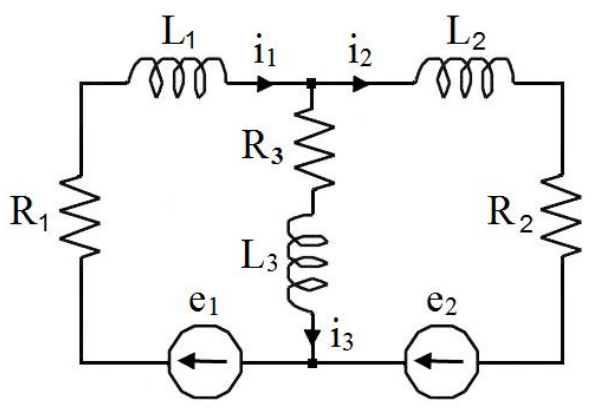

Fig. 4. Descriptor electrical circuit of Example 5

Using the Kirchhoff's laws we obtain the equations:

$e_{1}=R_{1} i_{1}+L_{1} \frac{d i_{1}}{d t}+R_{3} i_{3}+L_{3} \frac{d i_{3}}{d t}$,
$e_{2}=R_{2} i_{2}+L_{2} \frac{d i_{2}}{d t}-R_{3} i_{3}-L_{3} \frac{d i_{3}}{d t}$ which can be written in the form:

$E \frac{d}{d t}\left[\begin{array}{l}i_{1} \\ i_{2} \\ i_{3}\end{array}\right]=A\left[\begin{array}{l}i_{1} \\ i_{2} \\ i_{3}\end{array}\right]+B\left[\begin{array}{l}e_{1} \\ e_{2}\end{array}\right]$

where:

$\begin{aligned} E & =\left[\begin{array}{lll}L_{1} & 0 & L_{3} \\ 0 & L_{2} & -L_{3} \\ 0 & 0 & 0\end{array}\right], A=\left[\begin{array}{lll}-R_{1} & 0 & -R_{3} \\ 0 & -R_{2} & R_{3} \\ 1 & -1 & -1\end{array}\right], \\ B & =\left[\begin{array}{ll}1 & 0 \\ 0 & 1 \\ 0 & 0\end{array}\right] .\end{aligned}$

The condition (39) is satisfied since $\operatorname{det} E=0$ and:

$$
\begin{aligned}
& \operatorname{det}[E s-A]=\left|\begin{array}{lll}
R_{1}+s L_{1} & 0 & R_{3}+s L_{3} \\
0 & R_{2}+s L_{2} & -R_{3}-s L_{3} \\
-1 & 1 & 1
\end{array}\right| \\
& =\left[L_{1}\left(L_{2}+L_{3}\right)+L_{2} L_{3}\right] s^{2}+\left[L_{1} R_{3}+L_{3} R_{1}+L_{2}\left(R_{1}+R_{3}\right)\right. \\
& \left.+\left(L_{1}+L_{3}\right) R_{2}\right] s+R_{1} R_{3}+R_{2}\left(R_{1}+R_{2}\right) \neq 0 .
\end{aligned}
$$

Therefore, the characteristic equation of the electrical circuit has the form:

$S^{2}+\frac{L_{1} R_{3}+L_{3} R_{1}+L_{2}\left(R_{1}+R_{3}\right)+\left(L_{1}+L_{3}\right) R_{2}}{L_{1}\left(L_{2}+L_{3}\right)+L_{2} L_{3}} S$

$+\frac{R_{1} R_{3}+R_{2}\left(R_{1}+R_{2}\right)}{\left.L_{1}\left(L_{2}+L_{3}\right)+L_{2} L_{3}\right]}=0$

The descriptor electrical circuit shown in Fig. 4. is positive if $i_{k}(t) \geq 0, k=1,2,3$ for any initial conditions $i_{k}(0) \geq 0$, $k=1,2,3$ and all $e_{i}(t) \geq 0, \mathrm{i}=1,2$ for $t \geq 0$.

Substituting $i_{1}(t)=i_{2}(t)+i_{3}(t)$ into (40) we obtain:

$\left[\begin{array}{ll}L_{1} & L_{1}+L_{3} \\ L_{2} & -L_{3}\end{array}\right] \frac{d}{d t}\left[\begin{array}{l}i_{2} \\ i_{3}\end{array}\right]=\left[\begin{array}{ll}-R_{1} & -\left(R_{1}+R_{3}\right) \\ -R_{2} & R_{3}\end{array}\right]\left[\begin{array}{l}i_{2} \\ i_{3}\end{array}\right]+\left[\begin{array}{l}e_{1} \\ e_{2}\end{array}\right]$

and:

$\frac{d}{d t}\left[\begin{array}{l}i_{2} \\ i_{3}\end{array}\right]=\bar{A}\left[\begin{array}{l}i_{2} \\ i_{3}\end{array}\right]+\bar{B}\left[\begin{array}{l}e_{1} \\ e_{2}\end{array}\right]$

$\bar{B}=\left[\begin{array}{ll}L_{1} & L_{1}+L_{3} \\ L_{2} & -L_{3}\end{array}\right]^{-1}=\frac{1}{L_{1} L_{3}+L_{2}\left(L_{1}+L_{3}\right)}\left[\begin{array}{ll}L_{3} & L_{1}+L_{3} \\ L_{2} & -L_{1}\end{array}\right]$.

From (45b) it follows that $\bar{A} \in M_{2}$ if and only if $\left(R_{1}+\right.$ $\left.\mathrm{R}_{3}\right) \mathrm{L}_{2} \geq \mathrm{R}_{3} \mathrm{~L}_{1}$ and $\mathrm{R}_{1} \mathrm{~L}_{2} \geq \mathrm{R}_{2} \mathrm{~L}_{1}$, and $\overline{\mathrm{B}} \in \mathfrak{R}_{+}^{2 \times 2}$ if and only if $\mathrm{L}_{1}=0$.

Therefore, the descriptor electrical circuit is not positive for all values of the resistances $R_{k}, k=1,2,3$ and inductances $L_{k}$, $k=1,2,3$.

Using the mesh method to the electrical circuit we obtain

$Z(s)=\left[\begin{array}{ll}R_{1}+R_{3}+s\left(L_{1}+L_{3}\right) & -R_{3}-s L_{3} \\ -R_{3}-s L_{3} & R_{2}+R_{3}+s\left(L_{2}+L_{3}\right)\end{array}\right]$ 


$$
\begin{aligned}
& \operatorname{det} Z(s)=\left[R_{1}+R_{3}+s\left(L_{1}+L_{3}\right)\right]\left[R_{2}+R_{3}+s\left(L_{2}+L_{3}\right)\right] \\
& -\left(R_{3}+s L_{3}\right)^{2}=\left[L_{1}\left(L_{2}+L_{3}\right)+L_{2} L_{3}\right] s^{2} \\
& +\left[L_{1} R_{3}+L_{3} R_{1}+L_{2}\left(R_{1}+R_{3}\right)+\left(L_{1}+L_{3}\right) R_{2}\right] s \\
& +R_{1} R_{3}+R_{2}\left(R_{1}+R_{2}\right) .
\end{aligned}
$$

From comparison of (42) and (47) it follows that the characteristic equation obtained in the mesh method is identical with (43).

Using the node method to the electrical circuit we obtain:

$Y(s)=\frac{1}{R_{1}+s L_{1}}+\frac{1}{R_{2}+s L_{2}}+\frac{1}{R_{3}+s L_{3}}$

and:

$$
\begin{aligned}
& \operatorname{det} Y(s)=\frac{\left(R_{2}+s L_{2}\right)\left(R_{3}+s L_{3}\right)+\left(R_{1}+s L_{1}\right)\left(R_{3}+s L_{3}\right)+\left(R_{1}+s L_{1}\right)\left(R_{2}+s L_{2}\right)}{\left(R_{1}+s L_{1}\right)\left(R_{2}+s L_{2}\right)\left(R_{3}+s L_{3}\right)} \\
& =\frac{\left.L_{1}\left(L_{2}+L_{3}\right)+L_{2} L_{3}\right] s^{2}+\left[L_{1} R_{3}+L_{3} R_{1}+L_{2}\left(R_{1}+R_{3}\right)+\left(L_{1}+L_{3}\right) R_{2}\right] s}{\left(R_{1}+s L_{1}\right)\left(R_{2}+s L_{2}\right)\left(R_{3}+s L_{3}\right)} \\
& +\frac{R_{1} R_{3}+R_{2}\left(R_{1}+R_{2}\right)}{\left(R_{1}+s L_{1}\right)\left(R_{2}+s L_{2}\right)\left(R_{3}+s L_{3}\right)} .
\end{aligned}
$$

Therefore, the characteristic equation obtained in the node method is identical with (43).

In general case we have the following theorem.

Theorem 7. The characteristic equations of the descriptor linear electrical circuit composed of resistors, coils and capacitors obtained by the state space method, mesh method and node method are equivalent.

Proof. The proof is similar to the proof of Theorem 5.

\section{CHARACTERISTIC EQUATIONS OF FRACTIONAL ELECTRICAL CIRCUITS}

\subsection{State space method}

Consider the fractional linear electrical circuit described by (7) for $\mathrm{Bu}(\mathrm{t})=0$. Applying to the equation (7) the Laplace transform and taking into account that:

$\mathrm{L}\left[D^{\alpha} x(t)\right]=s^{\alpha} X(s)-s^{1-\alpha} x(0)$

for $x(0)=0$ we obtain:

$\left[I_{n} s^{\alpha}-A\right] X(s)=0$.

The characteristic polynomial of the electrical circuit (of matrix A) has the form:

$p\left(s^{\alpha}\right)=\operatorname{det}\left[I_{n} s^{\alpha}-A\right]$

$=\left(s^{\alpha}\right)^{n}+a_{n-1}\left(s^{\alpha}\right)^{n-1}+\ldots+a_{1} s^{\alpha}+a_{0}$

and its characteristic equation $p\left(s^{\alpha}\right)=0$.

Example 6. Consider the fractional electrical circuit shown in Fig. 5 with given resistances $R_{1}, R_{2}, R_{3}$, capacitances $C_{1}, C_{2}$ and source voltage $e$.

Using the Kirchhoff's laws we obtain the equations:

$e=R_{1} C_{1} \frac{d^{\alpha} u_{1}}{d t^{\alpha}}+u_{1}+R_{3}\left(C_{1} \frac{d^{\alpha} u_{1}}{d t^{\alpha}}+C_{2} \frac{d^{\alpha} u_{2}}{d t^{\alpha}}\right)$,

$e=+R_{3}\left(C_{1} \frac{d^{\alpha} u_{1}}{d t^{\alpha}}+C_{2} \frac{d^{\alpha} u_{2}}{d t^{\alpha}}\right)+u_{2}+R_{2} C_{2} \frac{d^{\alpha} u_{2}}{d t^{\alpha}}$

which can be written in the form:

$$
\begin{aligned}
& {\left[\begin{array}{ll}
\left(R_{1}+R_{3}\right) C_{1} & R_{3} C_{2} \\
R_{3} C_{1} & \left(R_{2}+R_{3}\right) C_{2}
\end{array}\right] \frac{d^{\alpha}}{d t^{\alpha}}\left[\begin{array}{l}
u_{1} \\
u_{2}
\end{array}\right]} \\
& =\left[\begin{array}{ll}
-1 & 0 \\
0 & -1
\end{array}\right]\left[\begin{array}{l}
u_{1} \\
u_{2}
\end{array}\right]+\left[\begin{array}{l}
1 \\
1
\end{array}\right] e
\end{aligned}
$$

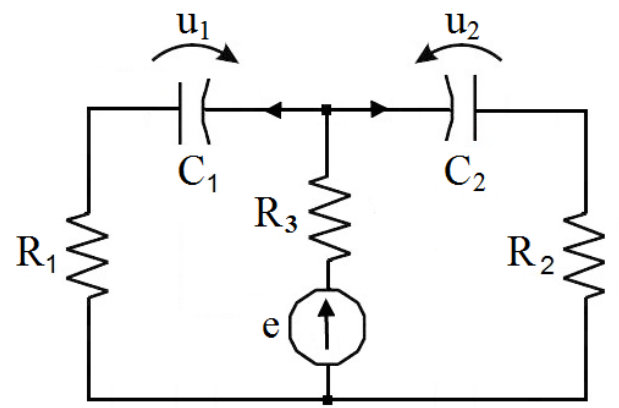

Fig. 5. Electrical circuit of Example 6

Premultiplying (54) by the inverse matrix:

$$
\begin{aligned}
& {\left[\begin{array}{ll}
\left(R_{1}+R_{3}\right) C_{1} & R_{3} C_{2} \\
R_{3} C_{1} & \left(R_{2}+R_{3}\right) C_{2}
\end{array}\right]^{-1} } \\
&= \frac{1}{\Delta}\left[\begin{array}{ll}
\left(R_{2}+R_{3}\right) C_{2} & -R_{3} C_{2} \\
-R_{3} C_{1} & \left(R_{1}+R_{3}\right) C_{1}
\end{array}\right], \\
& \Delta=\left[R_{1}\left(R_{2}+R_{3}\right)+R_{2} R_{3}\right] C_{1} C_{2}
\end{aligned}
$$

we obtain:

$\frac{d^{\alpha}}{d t^{\alpha}}\left[\begin{array}{l}u_{1} \\ u_{2}\end{array}\right]=A\left[\begin{array}{l}u_{1} \\ u_{2}\end{array}\right]+B e$,

where:

$$
\begin{gathered}
A=\left[\begin{array}{cc}
-\frac{R_{2}+R_{3}}{\left[R_{1}\left(R_{2}+R_{3}\right)+R_{2} R_{3}\right] C_{1}} & \frac{R_{3}}{\left[R_{1}\left(R_{2}+R_{3}\right)+R_{2} R_{3}\right] C_{1}} \\
\frac{R_{3}}{\left[R_{1}\left(R_{2}+R_{3}\right)+R_{2} R_{3}\right] C_{2}} & -\frac{R_{1}+R_{3}}{\left[R_{1}\left(R_{2}+R_{3}\right)+R_{2} R_{3}\right] C_{2}}
\end{array}\right], \\
B=\left[\begin{array}{c}
\frac{R_{2}}{\left[R_{1}\left(R_{2}+R_{3}\right)+R_{2} R_{3}\right] C_{1}} \\
\frac{R_{1}}{\left[R_{1}\left(R_{2}+R_{3}\right)+R_{2} R_{3}\right] C_{2}}
\end{array}\right] .
\end{gathered}
$$

From (56b) it follows that the fractional electrical circuit is positive since $A \in M_{2}$ and $B \in \Re_{+}^{2}$ for all nonzero values of the resistances and capacitances.

The characteristic equation of the matrix $A$ has the form:

$\operatorname{det}\left[I_{2} \lambda-A\right]$

$=\left[\begin{array}{cc}\lambda+\frac{R_{2}+R_{3}}{\left[R_{1}\left(R_{2}+R_{3}\right)+R_{2} R_{3}\right] C_{1}} & -\frac{R_{3}}{\left[R_{1}\left(R_{2}+R_{3}\right)+R_{2} R_{3}\right] C_{1}} \\ -\frac{R_{3}}{\left[R_{1}\left(R_{2}+R_{3}\right)+R_{2} R_{3}\right] C_{2}} & \lambda+\frac{R_{1}+R_{3}}{\left[R_{1}\left(R_{2}+R_{3}\right)+R_{2} R_{3}\right] C_{2}}\end{array}\right]$

$=\lambda^{2}+\frac{\left(R_{1}+R_{3}\right) C_{1}+\left(R_{2}+R_{3}\right) C_{2}}{\left[R_{1}\left(R_{2}+R_{3}\right)+R_{2} R_{3}\right] C_{1} C_{2}} \lambda+\frac{1}{\left[R_{1}\left(R_{2}+R_{3}\right)+R_{2} R_{3}\right] C_{1} C_{2}}=0$,

where $\lambda=s^{\alpha}$.

\subsection{Mesh method}

Using the mesh method and the Laplace transform for zero initial conditions the fractional linear electrical circuits in transient states can be described by the equation:

$Z(\lambda) X(\lambda)=E(\lambda), \lambda=s^{\alpha}$

where: $X(\lambda)=\mathrm{L}[x(t)]$ is the Laplace transform of the mesh currents. 
$Z(\lambda)=\left[\begin{array}{lll}Z_{11}(\lambda) & \cdots & Z_{1 n}(\lambda) \\ \vdots & \ddots & \vdots \\ Z_{n 1}(\lambda) & \cdots & Z_{n n}(\lambda)\end{array}\right], E(\lambda)=\left[\begin{array}{l}E_{1}(\lambda) \\ \vdots \\ E_{n}(\lambda)\end{array}\right]$.

Example 7. For the fractional electrical circuit shown in Fig. 5. using the mesh method and the Laplace transform for zero initial conditions we obtain:

$\left[\begin{array}{ll}R_{1}+R_{3}+\frac{1}{\lambda C_{1}} & R_{3} \\ R_{3} & R_{2}+R_{3}+\frac{1}{\lambda C_{2}}\end{array}\right]\left[\begin{array}{l}I_{1}(\lambda) \\ I_{2}(\lambda)\end{array}\right]=\left[\begin{array}{l}E(\lambda) \\ E(\lambda)\end{array}\right]$,

where: $I_{k}(\lambda)=\mathrm{L}\left[i_{k}(t)\right], E(\lambda)=\mathrm{L}[e(t)], k=1,2$.

In this case:

$Z(\lambda)=\left[\begin{array}{ll}R_{1}+R_{3}+\frac{1}{\lambda c_{1}} & R_{3} \\ R_{3} & R_{2}+R_{3}+\frac{1}{\lambda C_{2}}\end{array}\right]$

and:

$\operatorname{det} Z(\lambda)=\left|\begin{array}{ll}R_{1}+R_{3}+\frac{1}{\lambda C_{1}} & R_{3} \\ R_{3} & R_{2}+R_{3}+\frac{1}{\lambda C_{2}}\end{array}\right|$

$=\frac{\left[\left(R_{1}+R_{3}\right) \lambda C_{1}+1\right]\left[\left(R_{2}+R_{3}\right) \lambda C_{2}+1\right]-R_{3}^{2} C_{1} C_{2} \lambda^{2}}{\lambda^{2} C_{1} C_{2}}$

$=\left[\begin{array}{l}\lambda^{2}+\frac{\left(R_{1}+R_{3}\right) C_{1}+\left(R_{2}+R_{3}\right) C_{2}}{\left[R_{1}\left(R_{2}+R_{3}\right)+R_{2} R_{3}\right] C_{1} C_{2}} \lambda \\ +\frac{1}{\left[R_{1}\left(R_{2}+R_{3}\right)+R_{2} R_{3}\right] C_{1} C_{2}}\end{array}\right]$

$\times\left[R_{1}\left(R_{2}+R_{3}\right)+R_{2} R_{3}\right]=0$.

Dividing (61) by $R_{1}\left(R_{2}+R_{3}\right)+R_{2} R_{3}$ we obtain the characteristic equation (57). Therefore, $\operatorname{det}\left[\mathrm{I}_{2} \lambda-\mathrm{A}\right]=0$ and $\operatorname{det} Z(\lambda)=0$ represent the same characteristic equation of the fractional electrical circuit shown in Fig. 5.

\subsection{Node method}

Using the node method and the Laplace transform for zero initial conditions the fractional linear electrical circuits in transient states can be described by the equation:

$Y(\lambda) V(\lambda)=I_{z}(\lambda), \lambda=s^{\alpha}$

where:

$Y(\lambda)=\left[\begin{array}{lll}Y_{11}(\lambda) & \cdots & Y_{1 q}(\lambda) \\ \vdots & \ddots & \vdots \\ Y_{q 1}(\lambda) & \cdots & Y_{q q}(\lambda)\end{array}\right], V(\lambda)=\left[\begin{array}{l}V_{1}(\lambda) \\ \vdots \\ V_{q}(\lambda)\end{array}\right]$,

$I_{z}(\lambda)=\left[\begin{array}{l}I_{z 1}(\lambda) \\ \vdots \\ I_{z q}(\lambda)\end{array}\right]$,

$q$ is the number of linearly independent nodes, $Y_{i j}(\lambda)$ and $V_{i}(\lambda)$, $i, j=1, \ldots, q$ are Laplace transforms of conductances and current sources of the electrical circuit, respectively.

Example 8. For the fractional electrical circuit shown in Fig. 6 using the node method and the Laplace transform for zero initial conditions we obtain (62) with:

$Y(s)=Y_{11}(s)=\frac{1}{R_{1}+\frac{1}{\lambda C_{1}}}+\frac{1}{R_{2}+\frac{1}{\lambda C_{2}}}+\frac{1}{R_{3}}$

$=\frac{C_{1} \lambda}{1+R_{1} C_{1} \lambda}+\frac{C_{2} \lambda}{1+R_{2} C_{2} \lambda}+\frac{1}{R_{3}}$

and $I_{z}(\lambda)=\frac{E(\lambda)}{R_{3}}$.

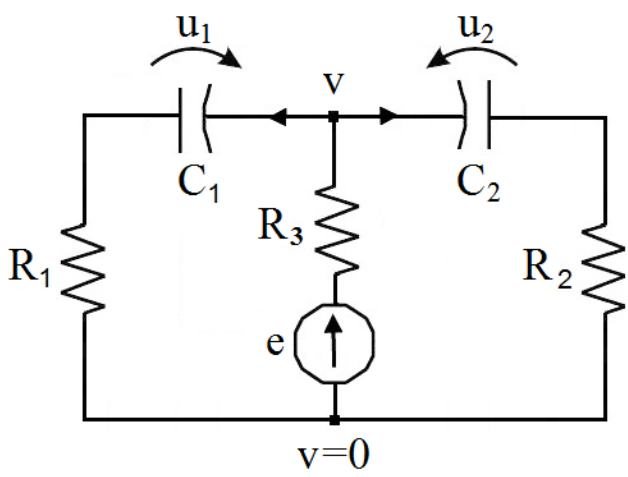

Fig. 6. Electrical circuit

From (63) we have:

$\operatorname{det} Y(\lambda)=$

$\frac{\lambda^{2}\left[R_{1}\left(R_{2}+R_{3}\right)+R_{2} R_{3}\right] C_{1} C_{2}+\lambda\left[\left(R_{1}+R_{3}\right) C_{1}+\left(R_{2}+R_{3}\right)\right] C_{2}+1}{\left(1+R_{1} C_{1} \lambda\right)\left(1+R_{2} C_{2} \lambda\right) R_{3}}$

$=0$

and:

$\lambda^{2}+\frac{\left(R_{1}+R_{3}\right) C_{1}+\left(R_{2}+R_{3}\right) C_{2}}{\left[R_{1}\left(R_{2}+R_{3}\right)+R_{2} R_{3}\right] C_{1} C_{2}} \lambda$

$+\frac{1}{\left[R_{1}\left(R_{2}+R_{3}\right)+R_{2} R_{3}\right] C_{1} C_{2}}=0$

Therefore, $\operatorname{det} Y(\lambda)=0$ the same characteristic equation of the fractional electrical circuit as the equation (57).

In general case we have the following theorem.

Theorem 8. The characteristic equation of any fractional linear electrical circuit in the state space method $\operatorname{det}\left[I_{n} \lambda-A\right]=0$, in the mesh method $\operatorname{det} Z(\lambda)=0$ and in the node method $\operatorname{det} Y(\lambda)=0$ is the same.

Proof. The proof is similar to the proof of Theorem 5.

The considerations presented in section 4 can be easily extended to the fractional descriptor linear electrical circuits.

\section{CONCLUDING REMARKS}

The problem of calculation of the characteristic equations of the standard positive and descriptor linear electrical circuits of integer and fractional orders has been addressed. It has been shown that the characteristic equations of standard and descriptor linear electrical circuits are independent of the method used in their analysis. The state space method, the mesh method and the node method have been analyzed (Theorems 5 and 8). The results obtained for standard linear electrical circuits can be extended to the fractional linear electrical circuits by substituting $s$ by $\lambda=s^{\alpha}$, where $\alpha$ is the order of the fractional differential equation. The considerations have been illustrated by examples of standard and descriptor linear electrical circuits.

\section{REFERENCES}

1. Antsaklis P.J., Michel A.N. (2006), Linear Systems, Birkhauser, Boston.

2. Cholewicki T. (1967), Theoretical Electrotechnics, WNT, Warszawa (in Polish). 
3. Dzieliński A., Sierociuk D., Sarwas G. (2009), Ultracapacitor parameters identification based on fractional order model, Proc. ECC'09, Budapest.

4. Farina L., Rinaldi S. (2000), Positive Linear Systems; Theory and Applications, J. Wiley, New York.

5. Kaczorek T. (1992), Linear Control Systems vol. 1, Research Studies Press, J. Wiley, New York.

6. Kaczorek T. (2002), Positive $1 D$ and 2D Systems, Springer-Verlag, London.

7. Kaczorek T. (2008a), Fractional positive continuous-time systems and their reachability, Int. J. Appl. Math. Comput. Sci., 18(2), 223-228

8. Kaczorek T. (2008b), Practical stability of positive fractional discretetime linear systems, Bull. Pol. Acad. Sci. Tech., 56(4), 313-317.

9. Kaczorek T. (2009), Asymptotic stability of positive fractional 2D linear systems, Bull. Pol. Acad. Sci. Tech., 57(3), 289-292.

10. Kaczorek T. (2010), Positive linear systems with different fractional orders, Bull. Pol. Acad. Sci. Techn., 58(3), 453-458.

11. Kaczorek T. (2011a), Positive linear systems consisting of $n$ subsystems with different fractional orders, IEEE Trans. Circuits and Systems, 58(6), 1203-1210.

12. Kaczorek T. (2011b), Positivity and reachability of fractional electrical circuits, Acta Mechanica et Automatica, 5(2), 42-51.

13. Kaczorek T. (2011c), Positive electrical circuits and their reachability, Archives of Electrical Engineering, 60(3), 283-301.

14. Kaczorek T. (2011d), Controllability and observability of linear electrical circuits, Electrical Review, 87(9a), 248-254.

15. Kaczorek T. (2012a), Selected Problems of Fractional Systems Theory, Springer-Verlag, Berlin.

16. Kaczorek T. (2012b), Positive unstable electrical circuits, Electrical Review, 88(5a), 187-192.

17. Kaczorek T. (2013a), Positive fractional linear electrical circuits. Proceedings of SPIE, 8903, Bellingham WA, USA, Art. № 3903-35.

18. Kaczorek T. (2013b), Constructability and observability of standard and positive electrical circuits, Electrical Review, 89(7), 132-136.

19. Kaczorek T. (2013c), Zeroing of state variables in descriptor electrical circuits by state-feedbacks, Electrical Review, 89(10), 200-203.

20. Kaczorek T. (2014a), Polynomial approach to fractional descriptor electrical circuits, Computational Models for Business and Engineering Domains - ITHEA, Rzeszow, Poland.

21. Kaczorek T. (2014b), Decoupling zeros of positive continuous-time linear systems and electrical circuit, Advances in Systems Science. Advances in Intelligent Systems and Computing, 240, 1-15.
22. Kaczorek T. (2015a), A class of positive and stable time-varying electrical circuits, Electrical Review, 91(5), 121-124.

23. Kaczorek T. (2015b), Normal positive electrical circuits, IET Circuits Theory and Applications, 9(5), 691-699.

24. Kaczorek T. (2016), Minimal-phase positive electrical circuits, Electrical Review, 92(3), 182-189.

25. Kaczorek T., Rogowski K. (2015), Fractional Linear Systems and Electrical Circuits, Studies in Systems, 13, Springer International Publishing.

26. Kailath T. (1980), Linear Systems, Prentice-Hall, Englewood Cliffs, New York.

27. Oldham K.B., Spanier J. (1974), The Fractional Calculus, Academic Press, New York.

28. Ostalczyk P. (2008), Epitome of the fractional calculus: Theory and its Applications in Automatics, Technical University of Łódź Press, Łódź (in Polish).

29. Ostalczyk P. (2016) Discrete Fractional Calculus: Selected Applications in Control and Image Processing, Series in Computer Vision, 4.

30. Podlubny I. (1999), Fractional Differential Equations, Academic Press, San Diego.

31. Rosenbrock H. (1970), State-Space and Multivariable Theory, J. Wiley, New York.

32. Sajewski Ł. (2016), Reachability, observability and minimum energy control of fractional positive continuous-time linear systems with two different fractional orders, Multidimensional Systems and Signal Processing, 27(1), 27-41.

33. Vinagre B.M., Monje C.A., Calderon A.J. (2002), Fractional order systems and fractional order control actions, Lecture 3 IEEE CDC'02 TW\#2: Fractional calculus Applications in Automatic Control and Robotics.

34. Wolovich W.A. (1974), Linear Multivariable Systems, SpringerVerlag, New York.

35. Żak S.H. (2003), Systems and Control, Oxford University Press, New York.

This work was supported by National Science Centre in Poland under work No. 2014/13/B/ST7/03467. 